\title{
MODAL MINERALOGY OF PLANETARY SURFACES FROM VISIBLE AND NEAR- INFRARED SPECTRAL DATA
}

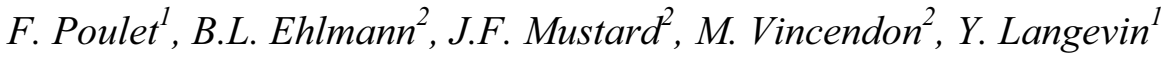 \\ ${ }^{1}$ Institut d'Astrophysique Spatiale, Université Paris Sud, Orsay, France \\ ${ }^{2}$ Dept. of Geological Science, Brown University, Providence, USA
}

\begin{abstract}
Real planetary surfaces are composed of several to many different minerals and ices. Deconvolving a reflectance spectrum to material abundance in an unambiguous way is difficult, because the spectra are complex nonlinear functions of grain size, abundance, and material opacity. Multiple scattering models can provide approximate solutions to the radiative transfer in a particulate medium.

The paper examines the different approaches which deal with the theory of radiative transfer on atmosphereless bodies. We present the relative merits of two scattering theories based on the equivalent slab model: the extensively used Hapke theory [1] and the Shkuratov theory [2]. The performances of the two models for determining mineral abundance in multicomponent mixtures are also evaluated using laboratory data. Finally, one application on real planetary surfaces will be shown.
\end{abstract}

Index Terms - radiative transfer, modeling, Hapke, Shkuratov, imaging spectrometer

\section{INTRODUCTION}

The objective of modeling the observed spectral reflectance of a planetary surface is to determine the composition (ices, minerals, metals, organic solids), the type of mixture (molecular, intimate, areal), the relative abundances of components, and approximate surface microstructures or grains sizes that reflect the incident sunlight in the visible and near-infrared spectral regions. Quantitative models also allow an evaluation of the surface temperature in some cases. Depending upon the opacity, dimensions, and microstructure (e.g., crystalline or amorphous) of the particles composing the surface, the depth of the surface layer sampled by scattered incident sunlight can be a few micrometers or a few centimeters. Surfaces composed of small, highly opaque particles (e.g., the lunar maria) are sampled to micrometer depths, while surfaces covered with large, transparent crystals (e.g., Triton, with its solid N2 surface) are probed to depths up to several $\mathrm{cm}$.

\section{RADIATIVE TRANSFER IN ROUGH, PARTICULATE SURFACES}

A large amount of work dealing with the theory of radiative transfer on atmosphereless bodies has appeared in the literature. The two major aspects of the problem involve solving the integral equation of radiative transfer and developing a statistical representation of the regolith individual particles to relate particle properties to scatterer properties. The basic integral equation of the radiative transfer problem can be solved in different ways. In general, it is done by considering a small elementary volume of the scattering medium wherein the individual particles have some "average" albedo and phase function. By itself, this method, defined as the integral equation method, only estimates the overall optical properties of the medium and provides no information on the size or composition of the particles. Different numerical methods have been used to solve the radiative transfer equation: the discrete ordinatemethod [3,4], the adding method [5,6], the two-stream method and approximation of $\mathrm{H}$-functions of Chandrasekhar [1], and Ambartsumian nonlinear integral equation resolution [7]. Because the measured brightness is not directly related to the microscopic quantities (optical constant, grain size) by the integral equation approach itself, but merely to the grain albedo and phase function, a grain property model is needed. The regolith or powdered sample is represented statistically as a collection of particles of the same or different sizes and different composition, as represented by their refractive indices at the appropriate wavelength. This scheme, called the statistical method, was first described by [8], who was one of the first to characterize an elementary volume by the single scattering albedo of an "average" particle.

The integral equation and statistical approaches are generally combined to perform spectral modelings of particulate regolith surfaces for planetary applications. They can be used at different levels, namely, the microscopic level, with the goal of determining the local mean radiative properties (actual regolith grain properties, i.e., diameter, refractive index), and the macroscopic level, with the goal of modeling the radiative transfer within either vertically stratified or laterally variable (areally mixed) surfaces. The 
local mean properties of scattering and absorption are determined by the statistical method, while the integral equation method gives the bidirectional reflectance of a medium composed of such particles. Such a combined technique allows the measured brightness to be related to the microscopic quantities.

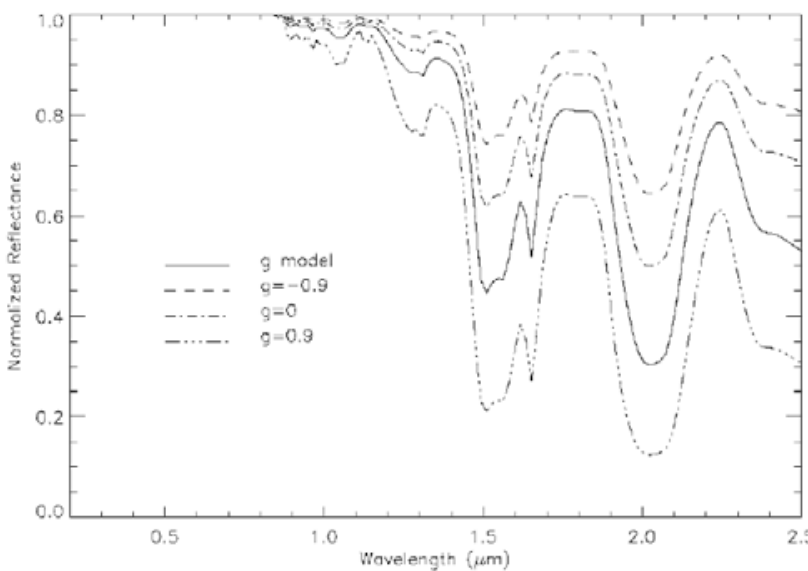

Fig. 1. This figure from [9] shows the dependence of the normalized reflectance of pure $\mathrm{H} 2 \mathrm{O}$ ice on $\mathrm{g}$ (upper panel). The label ' $g$ model' defines the spectrum calculated by using the Shkuratov model. The grain size is $10 \mu \mathrm{m}$.

The Hapke theory (H81 hereafter) was one of the first to derive the single scattering albedo for a grain of given size and refractive index and to provide this critical element in a simple and straightforward way. Because of this simplicity, $\mathrm{H} 81$ has become the dominant theory used in the planetary community. [2] (S99 hereafter) applied a similar form of statistical approach to calculate the albedo of a typical grain, but the approach after that point is different in one important regard. The principal difference between the Hapke and Shkuratov theories lies in the role of the phase function of individual regolith particles, where the scattering of sunlight occurs. In Hapke, the particle phase function is derived from best fits to reflectance data (over a wide range in phase angles), but comes into play only in the singly scattered light. The particle phase function thus derived is valid for surfaces of low albedo, where singlescattering dominates, and less valid for surfaces of high albedo in which multiple-scattering occurs; in Hapke's theory, the multiple-scattering term treats particles as isotropic scatterers. Most Solar System bodies studied with the Hapke theory show that the asymmetry parameter $g$ is negative. Fig. 1 [9] shows the result, with normalized spectra of surfaces made of pure water ice particles for different values of $g$. Most lab studies, however, suggest that regolith grains should be forward scattering, particularly in the $g=0$ limit normally assumed (no internal scattering). [10] used a Monte Carlo ray-tracing model to confirm that the phase function of irregular particles of size $>\lambda$ have strong forward-scattering lobes. The main difference between H81 and S99 is therefore the role of the phase function of individual particles of regolith, which is predicted (and generally forward directed) in the case of the Shkuratov model instead of being a free parameter as formulated in the Hapke model.

[9] also addressed the significant differences in the assumptions on the physical mixing in multicomponent surfaces, and the important effects the mixing has on the resulting synthetic spectra when compared with observations. For example, inhomogeneous particles that commonly occur in nature can take many forms, such as inclusions of one material in a host matrix of another material (intramixtures). There are several approaches to approximating the average dielectric constant of the combined media by using weighted averages of the dielectric constants of the various constituents. The most widely used are the models of Maxwell Garnett [11] and Bruggeman [12], and Bohren and Huffman [13].

\section{QUANTITATIVE EVALUATION OF LABORATORY MINERAL MIXTURES}

[14] presented applications of the spectral nonlinear spectral mixing method based on the Shkuratov model to invert spectra of controlled mineralogical samples. Four categories of samples have been used: simple and complex laboratory mixtures of mafic minerals; powdered and bulk samples of natural basalts. These tests indicate that the S99 spectral unmixing method can be used to determine the compositional and physical properties of a multi-component surface with similar confidence as previously existing methods: fractions are determined to better than $10 \%$ in most cases, similar to the MGM and Hapke model applied on the same samples. A noticeable advantage of the present method is to handle the components particle sizes as free parameters. Therefore the goodness of fit does not depend on a first guess of these parameters, in contrast to other methods. Besides, the estimates of particle size are in these tests accurate within a factor of 2 , which is enough to discriminate between large categories of grains and therefore between formation processes. Finally, the type of mixture involved (areal versus intimate) can be tested. In this regard, what appears to be the first successful modeling of basalt from mineral end-members is derived using another type of mixture (intramixtures) to simulate small oxides particles embedded in the plagioclase matrix.

An example of the performance of Hapke and Shkuratov models for determining phyllosilicate abundance in multicomponent mixtures is given Fig. 2. Because of the considerable compositional variability of phyllosilicates and 
changes in spectral properties related to hydration state, these alteration minerals may prove more challenging to model than mafic minerals. Model estimates of modal nontronite abundance in the nontronite-olivine mixtures are mostly within $10 \%$ of actual abundances (Fig. 2, middle panel). These uncertainties are similar to those inferred in the case of mafic mixtures [14]. Nontronite grain size is overestimated and olivine grain size is underestimated for the $30-70 \%$ nontronite-olivine mixtures. Future model runs will assess the efficacy of the Shkuratov model on other mixtures mixtures and compare these with the Hapke approach.
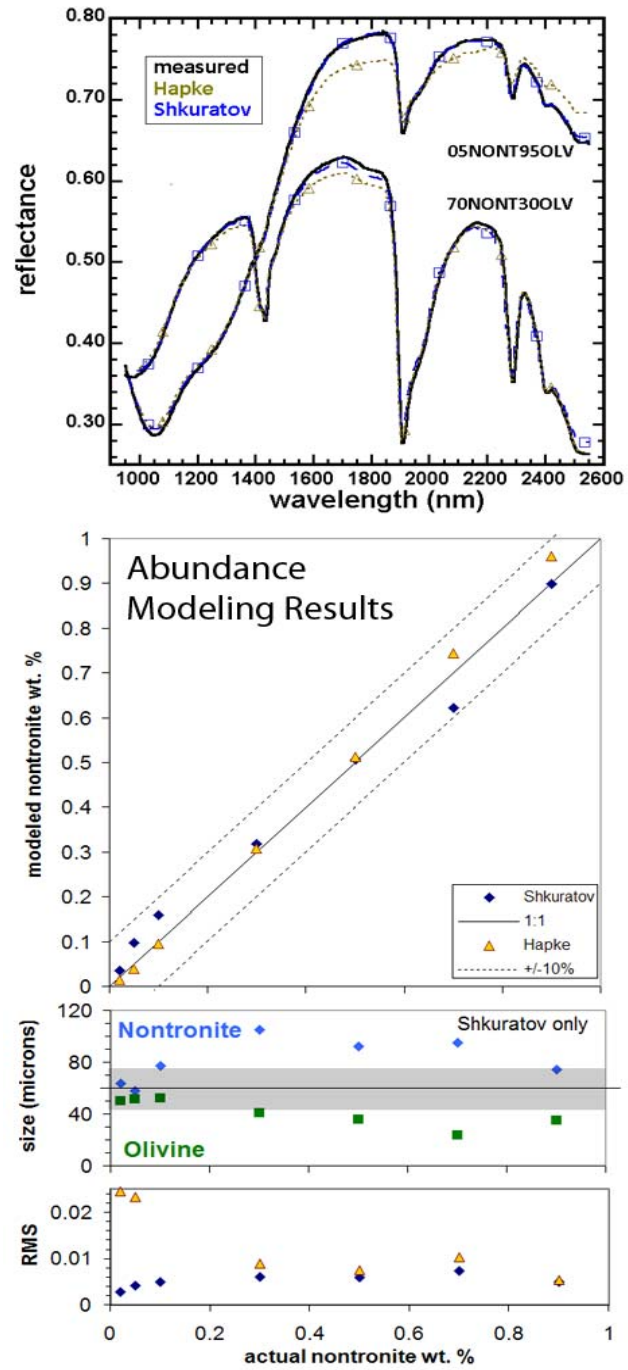

Fig. 2. (Top) Examples of the modeling of two laboratory mixtures (05NONT95OLV: $5 \%$ nontronite mixed with $95 \%$ of olivine, 70NONT30OLV: $30 \%$ nontronite mixed with $70 \%$ of olivine) using the Hapke and the Shkuratov models. (Middle) Calculated proportions of nontronite for seven mixtures of nontronite-olivine in comparison to its actual proportions. (Bottom) Calculated grain sizes for the two minerals and Residual Mean Squares (RMS) of the fit for the seven mixtures.

\section{APPLICATION}

We here show an example of the power of such an approach for constraining the mineralogy of a planetary surface, namely the mafic regions of Mars by using the The Observatoire pour la Minéralogie, l'Eau, les Glaces et l'Activité (OMEGA) instrument aboard the Mars Express spacecraft which began collecting visible and near-infrared $(0.4-5.1 \mu \mathrm{m})$ reflectance data from Mars in 2004. The derivation of the modal mineralogy of unaltered mafic-rich regions allows to evaluate constraints on the chemistry and petrology of the ancient crust, and to explore how crustal components might have arisen on Mars. OMEGA modal derived analyses [15] indicate a basaltic composition for all of the low albedo extended regions (Fig. 3). This modal mineralogy is typical of mafic magmatic rocks, which could be classified as gabbro-norites with minor amount of olivine for plutonic rocks; the volcanic equivalent nomenclature is two-pyroxene basalt. A decrease of the Low Calcium Pyroxene abundance through time is also observed [15]. The modal mineralogy of areas analyzed here clearly indicates an affinity with some of the basaltic Martian meteorites; they have young crystallization ages, suggesting that they are samples of young (dust-covered) volcanic centers such as Tharsis or Elysium. On the basis of a comparison of the OMEGA-based Mars surface composition with those of basaltic Shergottites, it appears that these meteorites could be witness of much older martian magmas.

\section{NEW DEVELOPPMENT}

Returning to the geometric optics issue, we note that both the Shkuratov and Hapke theories require for full validity that the scattering particles have dimensions substantially larger (at least a factor of 10) than the wavelength of the light. As the particle size approaches the wavelength of light, the theories are thus not directly applicable to measurements and must be used with care. In particular, the abundance of the fine-grained material may be underestimated because of a decrease in the spectral contrast as particle size decreases. Mustard and Hays [16] modeled the near-infrared spectra of samples having very fine particles using a combination of Mie theory to determine the single-scattering albedo, and a Hapke model to calculate the reflectance spectra. The results are qualitatively good, but extracting reliable quantitative information on the physical and compositional properties in this optical regime remains 
an issue. A limitation of the Mie-Hapke model is that the Mie solution explicitly assumes particles that are separated by several particle radii, which is clearly not the case in planetary regoliths. Particles with dimensions of the order of the wavelength of the light and smaller are predicted to scatter as Rayleigh particles, rather than as independent, forward-scattering Mie particles. Photometry of ultra-small particles of both low and high albedo over a range of phase angles is showing that as the particle size decreases there is no indication of a transition from a Mie to a Rayleigh scattering regime [17]. In addition, the development of the microscopic visible and NIR imaging spectrometer [18] implies to treat the light/grain interactions photon by photon. These new results and the new area of space exploration are prompting a reevaluation of the theory of scattering of light by planetary regoliths and/or a development of new models using Monte Carlo approach.

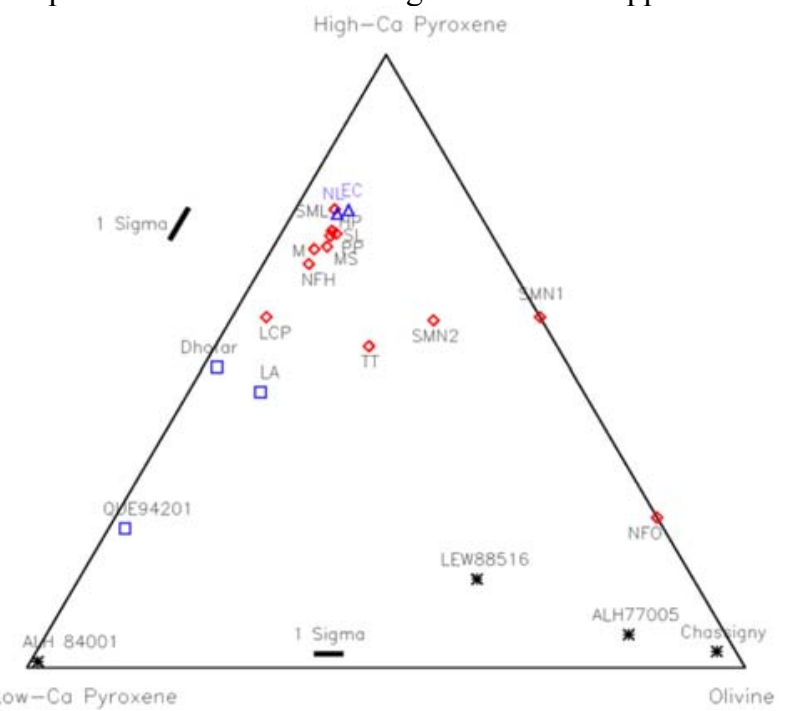

Fig. 3. Compositional distribution of old and mafic martian regions (red lozenges) projected onto the ternary diagram with High Calcium Pyroxene, Low Calcium Pyroxene and olivine as vertices. The Amazonian terrains are represented by blue triangles. The mineralogy of different martian meteorites (blue squares for basaltic Shergottites and black stars for other martian meteorites) are shown.

\section{REFERENCES}

[1] Hapke, B., Bidirectional reflectance spectroscopy. 1. Theory. J. Geophys. Res. 86, 3039-3054, 1981.

[2] Y. Shkuratov, L. Starukhina, H. Hoffmann, and G. Arnold, A model of spectral albedo of particulate surfaces: Implications for optical properties of the Moon. Icarus 137, 235-246, 1999.

[3] Chandrasekhar, S., Radiative Transfer. Dover, New York, 1960 .

[4] K. Stamnes, S.-C. Tsay, W. Wiscombe, and K. Jayaweera, Numerically stable algorithm for discrete-ordinate-method radiative transfer in multiple scattering and emitting layered media. Appl. Opt. 27, 2502-2509, 1988.

[5] Van de Hulst, H.C., Multiple Light Scattering. Academic Press, NewYork, 1980.

[6] J.F. de Haan, P.B. Bosma, and J.W. Hovenier, The adding method for multiple scattering calculations of polarized light. Astron. Astrophys. 183, 371-391, 1987.

[7] M.I. Mishchenko, Z.H.M. Dlugach, E.G. Yanovitskij, and N.T. Zakharova, Bidirectional reflectance of flat, otically thick particulate layers: An efficient radiative transfer solution and applications to snow and oil surfaces. J. Quant. Spectrosc. Radiat. Trans. 63, 409-432, 1999.

[8] N. Melamed, Optical properties of powders. J. Appl. Phys. 34, 560-570, 1983.

[9] F. Poulet, J.N. Cuzzi, D.P. Cruikshank, T. Roush, C.M. Dalle Ore, C.M., Comparison between the Shkuratov and Hapke scattering theories for solid planetary surfaces: Application to the surface composition of two Centaurs. Icarus 160, 313-324, 2002.

[10] W.M. Grundy, S. Douté, B. Schmitt, A Monte Carlo raytracing model for scattering and polarization by large particles with complex shapes, J. Geophys. Res. 105, 29290-29314, 2000.

[11] J.C. Maxwell Garnett, Colours in metal glasses and in metallic films, Phil. Trans. R. Soc. London A 203, 385-420, 1904.

[12] D.A.G. Bruggeman, Berechnung verschiedener physikalischer Konstanten von heterogenen Substanzen I. Dielektrizitätskonstanten und Leitfähighkeiten der Mischkörper aus isotropen Substanzen, Ann. Phys. (Leipzig) 24, 636 679, 1935. [13] Bohren, C.F., and D.R. Huffman, Absorption and Scattering of Light by Small Particles, Wiley, New York, 1983.

[14] F. Poulet, and S. Erard 2004. Nonlinear spectral mixing: Quantitative analysis of laboratory mineral mixtures. J. Geophys. Res. 109 (E2), E02009, 2004.

[15] Poulet F. et al., Quantitative compositional analysis of martian mafic regions using the MEx/OMEGA reflectance data. 2. Petrological implications, Icarus 201, 84-101, 2009.

[16] J.F. Mustard and J.E. Hays, Effects of hyperfine particles on reflectance spectra from 0.3 to 25 microns, Icarus $125,145-163$, 1997.

[17] J.L. Piatek, B. Hapke, R.M. Nelson, A.S. Hale, and W.D. Smythe, Size-dependent measurements of the scattering properties of planetary regolith analogs: A challenge to theory, Lunar Planet. Sci. XXXIV, abstract 1440, 2003.

[18] V. Leroi, J.-P. Bibring, and M. Berthe, Micromega/IR: Design and status of a near-infrared spectral microscope for in situ analysis of Mars samples, Planetary Space Science 57, 1068-1075, 2009. 